\title{
Review: comprehensive discharge planning plus post-discharge support reduces total readmissions in older patients with congestive heart failure
}

Phillips CO, Wright SM, Kern DE, et al. Comprehensive discharge planning with postdischarge support for older patients with congestive heart failure: a meta-analysis. JAMA 2004;291:1358-67.

\section{$Q$ In older patients with congestive heart failure (CHF), what is the effectiveness of comprehensive discharge planning plus post-discharge support on readmissions, all cause mortality, initial length of stay (LOS), quality of life (QOL), and overall medical costs?}

\section{METHODS}

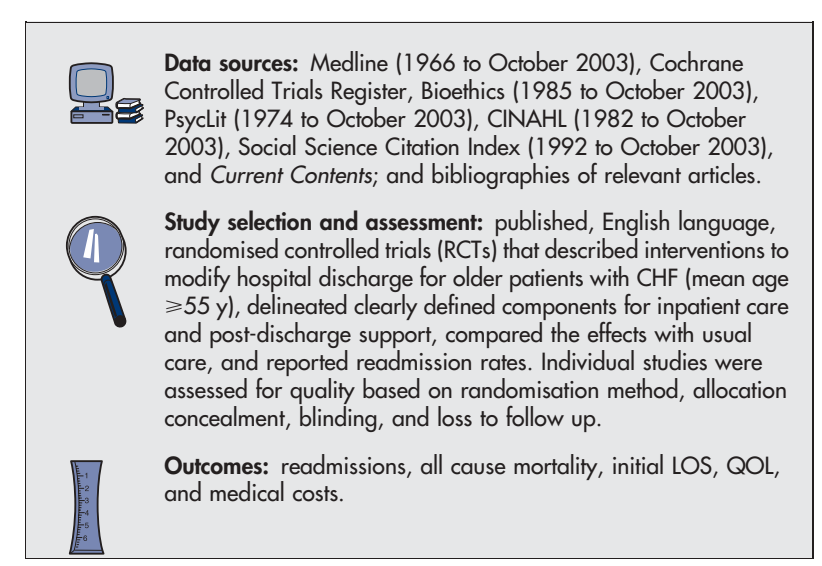

\section{MAIN RESULTS}

18 RCTs ( $\mathrm{n}=3304,62 \%$ men) done in 8 countries met the inclusion criteria. In 16 studies, mean age of patients was $\geqslant 70 \mathrm{y}$. Postdischarge support varied in duration and components, which included home visits, telephone contacts, clinic visits, continuous multidisciplinary home care, and day hospital care. Follow up ranged from 3-12 months (mean 8 mo). Comprehensive discharge planning plus post-discharge support reduced total readmissions, CHF or cardiovascular disease specific readmissions, and the combined endpoint of death or readmission compared with usual care, but not all cause mortality (table) or initial LOS (mean $8.4 \vee 8.5 \mathrm{~d}$, $\mathrm{p}=0.6$ ). Patients in the intervention group also had a greater percentage improvement in QOL scores $(26 \% v 14 \%, \mathrm{p}=0.01)$ and had similar or lower medical care costs per patient per month for initial LOS, intervention administration, outpatient care, and readmission.

For correspondence: $\operatorname{Dr} \mathrm{C}$ O Phillips, Johns Hopkins University School of Medicine, Baltimore, MD, USA. chr_phi@yahoo.com

Sources of funding: in part, National Heart, Lung, and Blood Institute.

\section{CONCLUSION}

In older patients with congestive heart failure, comprehensive discharge planning plus post-discharge support reduces total readmission rates and quality of life but not all cause mortality or initial length of stay, and has similar or lower medical costs.

\section{Commentary}

HF is an increasingly prevalent condition that is associated with high rates of hospital readmission, decreased QOL, and increased healthcare costs. ${ }^{1}$ Recent research has focused on exploring the effectiveness of different clinical service interventions to decrease hospital readmissions and hospital bed days and increase QOL.

The meta-analysis by Phillips et al provides a systematic assessment of studies that compared various discharge planning and post-discharge supports for older patients with CHF. They report an extensive search of published research, although other sources, such as unpublished studies and hand searches of bibliographies, were not included. Other strengths of the meta-analysis include the use of randomised trials with $\geqslant 100$ patients, data extraction by 2 reviewers using preset criteria, documentation of the size and precision of treatment effects, and use of appropriate tests of heterogeneity. All of the studies included some combination of discharge planning (generally related to medication counselling and homecare planning) with post-discharge support (telephone calls, home visits, or both) for 3-12 months. This rigorous analysis confirms the importance of careful discharge planning focused on education about medications, diet and self monitoring, and plans for follow up in the community, which should be standard components of nursing care provided to all patients leaving hospital. Similarly, the post-discharge supports of telephone calls and home visits should be considered within the realm of usual quality care. Phillips et al also remind us that these effective pre-discharge and post-discharge activities must be additional to the optimisation of medical treatment for CHF.

The meta-analysis by Phillips et al provides good evidence that patients with CHF should receive information about their medications and the signs and symptoms of worsening heart failure before discharge. Comprehensive discharge planning combined with careful follow up should result in fewer hospital admissions and increased quality of life for patients with CHF.

Elizabeth Rideout, RN, PhD School of Nursing, McMaster University Hamilton, Ontario, Canada

1 Taylor SJC, Underwood M, Parsons S, et al. Clinical service organization for heart failure (Protocol). Cochrane Database Syst Rev 2004;(2):CD002752.

Comprehensive discharge planning plus post-discharge support (intervention) $v$ usual care for older patients with $\mathrm{CHF}^{*}$

\begin{tabular}{lllll}
\hline & \multicolumn{2}{c}{ Pooled event rates } & & \\
\cline { 2 - 3 } Outcomes at a mean of 8 months & Intervention & Usual care & RRR (95\% Cl) & NNT (CI) \\
\hline Readmission & $35 \%$ & $43 \%$ & $19 \%(12$ to 26$)$ & $12(9$ to 20$)$ \\
CHF or CVD specific readmission & $23 \%$ & $33 \%$ & $32 \%(18$ to 44$)$ & 10 (7) 19$)$ \\
Death or readmission & $44 \%$ & $60 \%$ & $27 \%(18$ to 35$)$ & 7 (5 to 10$)$ \\
All cause mortality & $14 \%$ & $17 \%$ & $14 \%(-2$ to 28$)$ & Not significant \\
\hline
\end{tabular}

${ }^{*} \mathrm{CHF}=$ congestive heart failure, $\mathrm{CVD}=$ cardiovascular disease; other abbreviations defined in glossary. RRR, NNT, and Cl calculated from data in article. 